\title{
Chasing COVID-19 chemotherapeutics without putting the cart before the horse
}

\author{
Steven Rannard ${ }^{1}$, Thomas McDonald ${ }^{1}$, and Andrew Owen ${ }^{1}$ \\ ${ }^{1}$ University of Liverpool
}

July 7, 2020

Dear editor,

Given time, drug discovery programmes will undoubtedly yield highly potent drugs to form the basis of optimised COVID-19 regimens. However, if efficacious therapies can be identified from current medicines, repurposing represents the fastest route to establish deployable interventions and buy time for vaccine and novel drug development. It is important to note that effective medicines were rigorously optimised for the treatment of specific indications. Route of administration, dosage and schedules for existing therapies were optimised to provide adequate plasma/tissue pharmacokinetics and safety for their target disease or condition. These cannot be assumed to be optimal for COVID-19 but are often highly predictable from pre-existing data and clinical experience. For example, hydroxychloroquine and lopinavir/ritonavir recently failed to deliver benefits in RCTs for mild/moderate and severe disease, ${ }^{1,2}$ but the clear disconnect between reportedin vitro antiviral activity and known human pharmacokinetics after administration of approved doses was predictable. ${ }^{3}$

Interpretation of laboratory-based antiviral activity assessments is complicated by current uncertainty regarding the appropriateness of the existing model systems. The majority of in vitro antiviral screening assays have utilised Vero cells, which were derived from the kidney of African Green Monkey in the 1970s, and the lack of clinical evidence for which to validate the exposure-response relationship in humans is problematic. Evidence is emerging that the anti-SARS-CoV-2 activity of drugs may be higher in cells derived from humans. However, the question of which cell types are most representative of in vivo performance is yet to be addressed, and all that can really be concluded from current knowledge is that the susceptibility of SARS-CoV-2 to antivirals is cell-type-dependent. The consequences of this in terms of the variety of cell types known to be infected and/or sustain productive infection in vivo is equally uncertain, and further exacerbated by the lack of robustly validated animal models. However, repurposed drugs cannot be assumed to be active against SARS-CoV-2 at a dose that was optimised on the basis of potency for and accumulation at their initial therapeutic target.

Nucleoside/nucleotide polymerase inhibitors have proven highly successful for other viruses, but usually require combination with another drug class. Remdesivir and favipiravir have in vitro anti-SARS-CoV2 activity across multiple studies, and the unprecedented speed at which they have transitioned through COVID-19 RCTs can only be commended. ${ }^{4,5,6}$ Daily IV infusion may make inherent sense for severely ill patients, but a transformational impact for COVID-19 can only be realised if wide compatibility with global healthcare systems and equitable access across all country contexts is achieved. While reduction in symptom duration may mitigate healthcare saturation in high-income countries, the absence of a clear benefit for mortality diminishes game-changing potential. However, the clinical validation of the antiviral activity of such drugs will make them clear candidates for implementation as part of community-based interventions if other challenges are addressed. Importantly, the combination of nucleoside analogues with a secondary target such as the protease has stood the test of time in antiviral pharmacology. The recent 
reports of low-dose dexamethasone leading to an impact on mortality ${ }^{7}$ is a significant step forward but longterm mitigation of viral transmission, with subsequent economic and social restrictions, requires antiviral treatment or prevention to minimise hospitalisation through a community-targeted approach.

Focussing on existing single drugs, and not appropriately formulated medicines, will require the rethinking of a number of medicine development parameters such as posology, reformulation and therapeutic index (Figure 1); current HIV medicines, for example, are formulated for chronic (life-long) dosing to moderate and control disease but a successful COVID-19 therapy will likely require only a short term acute administration to rapidly cure the patient. Conversely, different considerations are required for longer-term applications in COVID-19 chemoprophylaxis, which could have a dramatic effect on control of the pandemic.

Many advanced drug delivery technologies have emerged in recent years. Long-acting drug delivery involving injectable, implantable or microarray patch mediated delivery have attracted enormous recent interest for prevention of other infectious diseases, ${ }^{8,9}$ and the ability to deliver potent antiviral combinations for a period of months could play a transformational role in the absence of a safe and efficacious vaccine. The physicochemistry and activity of the polymerase inhibitors, and other drugs with known anti SARS-Cov-2 activity, also warrants investigation of pulmonary delivery via nebuliser or metered dose inhaler for direct dosing to the upper airways to supplement systemic drug delivery as pre- or post-exposure prophylaxis. Several advanced drug delivery strategies can be applied rapidly and do not need to be prohibitively expensive for global community programmes. It seems unlikely that a global pandemic can be ended if effective medicines are only available to the few and equitable access is therefore of benefit to all. Importantly, relying solely upon pre-existing formulations and posologies optimised for other diseases carries inherent risk of rejecting drug candidates with an otherwise high potential for global impact.

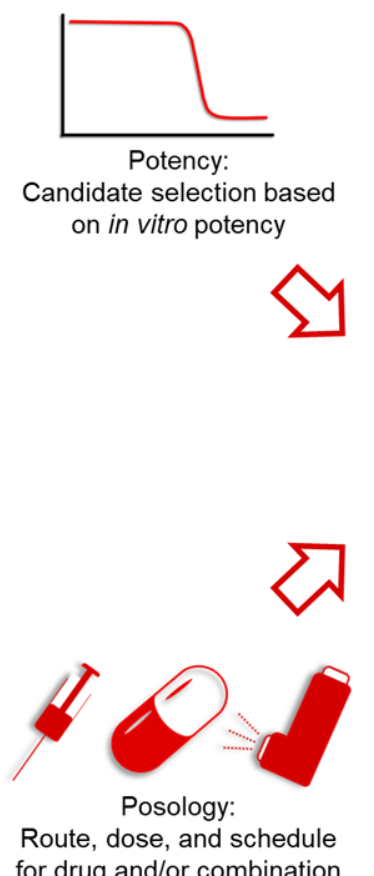

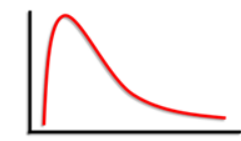

Plasma PK: Indication-specific therapeutic index
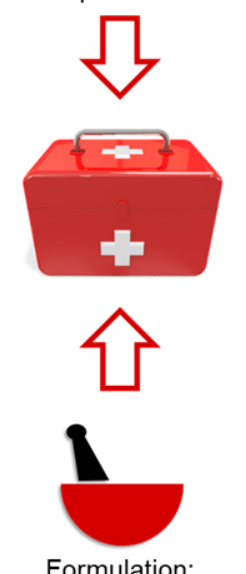

Route- and indicationspecific compatibility

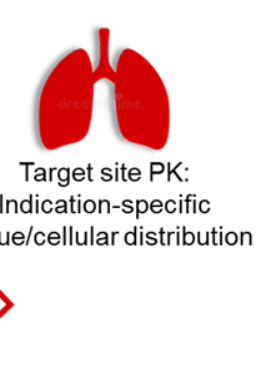

Successful medicine

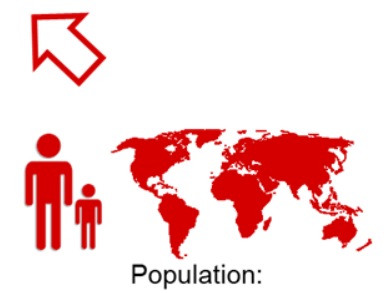

Inter- / intra-patient variability and country context

Figure 1 - Schematic representation of key medicine development considerations that need to be reconsidered when repurposing existing drug compounds

\section{References}

1. Tang W, Cao Z, Han M, et al. Hydroxychloroquine in patients with mainly mild to moderate coronavirus disease 2019: open label, randomised controlled trial. BMJ 2020; 369 : m1849. doi: 10.1136/bmj.m1849. 
2. Cao B, Wang Y, Wen D, et al. A Trial of Lopinavir-Ritonavir in Adults Hospitalized with Severe Covid-19. N Engl J Med 2020;382 (19): 1787-1799. doi: 10.1056/NEJMoa2001282.

3. Arshad U, Pertinez H, Box H, et al. Prioritisation of Anti-SARS-Cov-2 Drug Repurposing Opportunities Based on Plasma and Target Site Concentrations Derived from their Established Human Pharmacokinetics. Clin Pharmacol Ther 2020. doi: 10.1002/cpt.1909.

4. Wang Y, Zhang D, Du G, et al. Remdesivir in adults with severe COVID-19: a randomised, doubleblind, placebo-controlled, multicentre trial. Lancet 2020; 395 (10236): 1569-1578. doi: 10.1016/S01406736(20)31022-9.

5. Beigel JH, Tomashek KM, Dodd LE, et al. Remdesivir for the Treatment of Covid-19 - Preliminary Report. N Engl J Med 2020. DOI: 10.1056/NEJMoa2007764

6. Chen C, Zhang Y, Huang J, et al. Favipiravir versus Arbidol for COVID-19: A Randomized Clinical Trial. MedRxiv. 2020. doi: https://doi.org/10.1101/2020.03.17.20037432

7. Ledford H, Coronavirus breakthrough: dexamethasone is first drug shown to save lives Nature 2020 doi: 10.1038/d41586-020-01824-5

8. Bakshi RP, Tatham LM, Savage AC, et al. Long-acting injectable atovaquone nanomedicines for malaria prophylaxis. Nat Commun2018; 9 (1): 315. doi: 10.1038/s41467-017-02603-z.

9. Markowitz M, Frank I, Grant RM, et al. Safety and tolerability of long-acting cabotegravir injections in HIV-uninfected men (ECLAIR): a multicentre, double-blind, randomised, placebo-controlled, phase 2a trial. Lancet HIV 2017; 4 (8): e331-e340. doi: 10.1016/S2352-3018(17)30068-1.

\section{Figure Legend}

Figure: Effective redeployment of existing medicines requires explicit consideration of indication-specific factors relating to differences in potency, plasma and tissue pharmacokinetics (PK), safety, formulation, posology and barriers to equitable deployment across relevant populations and country contexts. 